\title{
Ionic Current Correlations Underlie the Global Tuning of Large Numbers of Neuronal Activity Attributes
}

\author{
Shunbing Zhao ${ }^{1}$ and Jorge Golowasch ${ }^{1,2}$ \\ ${ }^{1}$ Federated Department of Biological Sciences, New Jersey Institute of Technology and Rutgers University, Newark, New Jersey 07102, and ${ }^{2}$ Department of \\ Mathematical Sciences, New Jersey Institute of Technology, Newark, New Jersey 07102
}

Ionic conductances in identified neurons are highly variable. This poses the crucial question of how such neurons can produce stable activity. Coexpression of ionic currents has been observed in an increasing number of neurons in different systems, suggesting that the coregulation of ionic channel expression, by thus linking their variability, may enable neurons to maintain relatively constant neuronal activity as suggested by a number of recent theoretical studies. We examine this hypothesis experimentally using the voltage- and dynamic-clamp techniques to first measure and then modify the ionic conductance levels of three currents in identified neurons of the crab pyloric network. We quantify activity by measuring 10 different attributes (oscillation period, spiking frequency, etc.), and find linear, positive and negative relationships between conductance pairs and triplets that can enable pyloric neurons to maintain activity attributes invariant. Consistent with experimental observations, some of the features most tightly regulated appear to be phase relationships of bursting activity. We conclude that covariation (and probably a tightly controlled coregulation) of ionic conductances can help neurons maintain certain attributes of neuronal activity invariant while at the same time allowing conductances to change over wide ranges in response to internal or environmental inputs and perturbations. Our results also show that neurons can tune neuronal activity globally via coordinate expression of ion currents.

\section{Introduction}

Given that typically more than one ionic current determines the properties of any given neuronal activity attribute (spiking frequency, oscillation period, etc.), neurons may have mechanisms in place to ensure the coordinated regulation of currents controlling individual attributes. However, it is commonly assumed that individual ionic currents independently control distinct neuronal activity attributes even if most authors may implicitly recognize that more than one current affects the same attribute. Correlations among ionic conductances have now been measured in several systems (Linsdell and Moody, 1994; MacLean et al., 2003; Vähäsöyrinki et al., 2006; Khorkova and Golowasch, 2007; Peng and Wu, 2007; Tobin et al., 2009; Bergquist et al., 2010; Cao and Oertel, 2011; Amendola et al., 2012), suggesting that coregulation of ion channels exists at some level between transcription and membrane expression. Modeling and experimental studies have suggested that a possible functional role for the coexpression of ionic conductances is to ensure the invariance of the activity attributes they determine (Burdakov, 2005; MacLean et al., 2005; Swensen and Bean, 2005; Khorkova and Golowasch, 2007; Olypher and Calabrese, 2007; Hudson and

Received Dec. 29, 2011; revised July 17, 2012; accepted July 20, 2012.

Author contributions: J.G. designed research; S.Z. performed research; S.Z. and J.G. analyzed data; S.Z. and J.G. wrote the paper.

This study was funded by NIMH Grant 64711 to J.G. We thank Drs. Farzan Nadim, Amithabha Bose, Gareth Russell, and Victor Matveev for their helpful comments.

Correspondence should be addressed to Jorge Golowasch, 435 Colton Hall, Federated Department of Biological Sciences, New Jersey Institute of Technology, University Heights, Newark, NJ 07102. E-mail: Golowasch@njit.edu. DOI:10.1523/JNEUROSCI.6500-11.2012

Copyright $\odot 2012$ the authors $\quad 0270-6474 / 12 / 3213380-09 \$ 15.00 / 0$
Prinz, 2010; Amendola et al., 2012). This may be crucial in light of the high variability observed in the expression of ionic conductance levels in identified neurons across individuals (Golowasch et al., 1999; Schulz et al., 2006; Khorkova and Golowasch, 2007; Olypher and Calabrese, 2007; Temporal et al., 2012). Interestingly, so far correlations that can adequately be described by linear functions have been observed experimentally, almost all of which show positive slopes (Khorkova and Golowasch, 2007; Schulz et al., 2007; Tobin et al., 2009; Cao and Oertel, 2011; Amendola et al., 2012; but see Ransdell et al., 2012), although theoretical studies demonstrate that negative (or nonlinear) relationships are equally possible (Golowasch et al., 2002; Burdakov, 2005; Olypher and Calabrese, 2007; Taylor et al., 2009). Nevertheless, the role of correlated expression of multiple currents remains unknown and is the focus of this study.

Much of what is known about the correlated expression of ionic currents comes from experimental and theoretical work on the pyloric network of decapod crustaceans (MacLean et al., 2003; MacLean et al., 2005; Schulz et al., 2006, 2007; Khorkova and Golowasch, 2007; Hudson and Prinz, 2010). The pyloric network is known to maintain phase relationships of bursting activity between neurons nearly constant (Bucher et al., 2005; Goaillard et al., 2009), but no explanation has been found for this phenomenon. We hypothesize that phase invariance is partly due to the correlated expression of ionic currents. Here we use the dynamic clamp technique to ask what possible role the linearly correlated expression of three ionic currents $\left(I_{\mathrm{A}}, I_{\mathrm{H}}\right.$, and $\left.I_{\mathrm{HTK}}\right)$ in identified pyloric dilator (PD) neurons from the crab Cancer borealis plays in the normal behavior of this neuron. We find that simultaneously varying these three currents produces changes in 

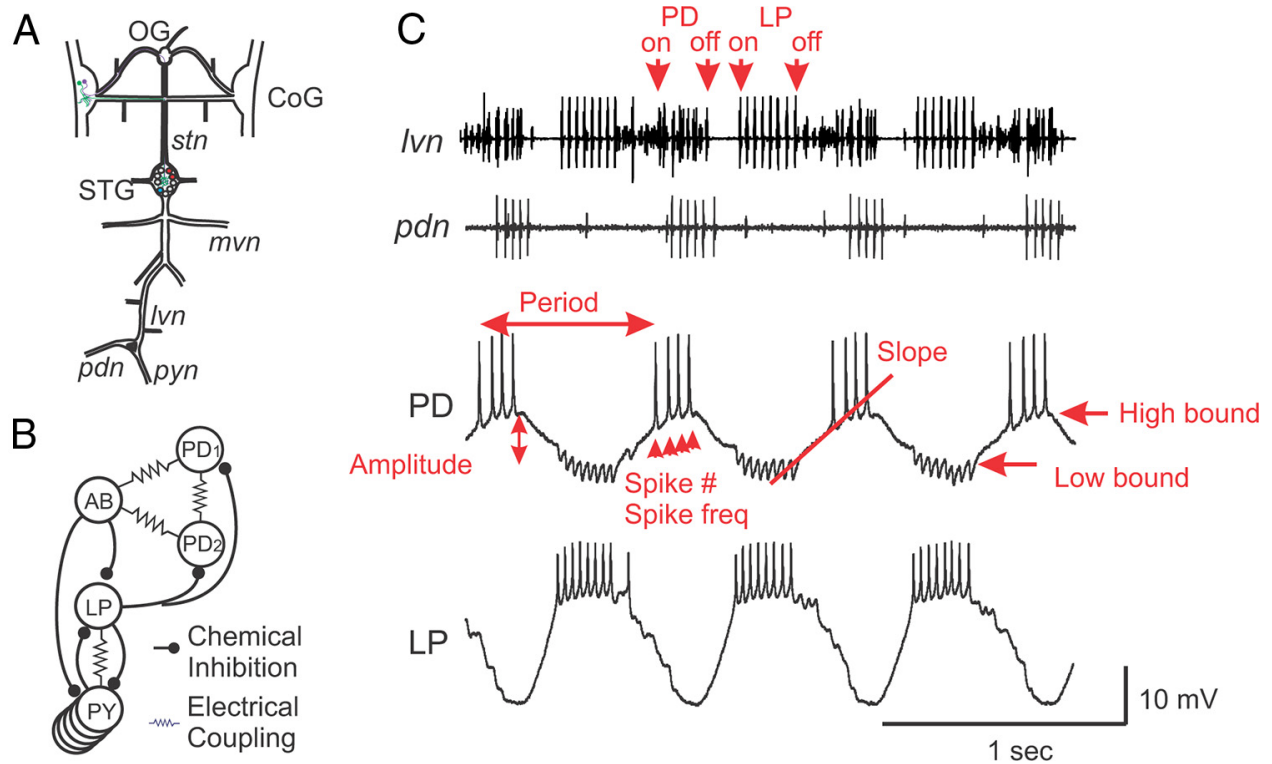

Figure 1. STNS and pyloric network activity. $A$, Schematic diagram of the STNS. The paired CoGs and OG contain modulatory projection neurons (colored cells) that send axons to the STG through the stomatogastric nerve (stn). Most pyloric neurons are motor neurons that project their axons via individual nerves [pyloric dilator nerve ( $p d n)$, pyloric constrictor nerve ( $p y n)$ shown] to their target muscles via the lateral ventricular nerve (Ivn) or through the medial ventricular nerve (mvn). B, Schematic diagram of the core of the pyloric network. The anterior burster (AB) and both PD neurons are all strongly electrically coupled to each other. LP receives inhibition from AB and inhibits both PD neurons, while it also reciprocally inhibits the PY neurons. $C$, The triphasic pyloric rhythm was recorded simultaneously from the lvn and pdn and intracellularly from one of the two PD neurons and the LP neuron. The arrows above the lvn recording highlight the onset (on) and termination (off) of the PD and LP neuron bursts. Red lines and arrows point out the other neuronal activity attributes measured in this study. IPSPs in PD are elicited by the action potentials of the LP neuron.

all the attributes of activity measured. These changes are related to the three currents in single, pairwise, or three-way combinations, defining linear relations among these currents that maintain the attribute values invariant across a large range of conductances.

\section{Materials and Methods}

Preparation and identification of the neurons. Experiments were conducted on the stomatogastric nervous system (STNS) of male crabs, Cancer borealis. Animals were obtained from local markets (Newark, NJ) and maintained in seawater tanks at $7-10^{\circ} \mathrm{C}$. The STNS was dissected out under a stereoscope and the complete isolated STNS, including the stomatogastric ganglion (STG), the esophageal ganglion (OG), the paired commissural ganglia $(\mathrm{CoG})$, and connecting nerves and motor nerves (Fig. 1 A; Selverston et al., 1976), were pinned down on a Sylgard-coated Petri dish. The STG was desheathed to enable electrode penetration of the pyloric neuron cell bodies. All preparations were continuously superfused with chilled $\left(10-13^{\circ} \mathrm{C}\right)$ physiological Cancer saline containing (in mм) $11 \mathrm{KCl}, 440 \mathrm{NaCl}, 13 \mathrm{CaCl}_{2}, 26 \mathrm{MgCl}_{2}, 11.2$ Trizma base, 5.1 maleic acid, $\mathrm{pH}$ 7.4-7.5.

Extracellular recordings from identified motor nerves (Fig. $1 A$ ) were made using stainless steel wire electrodes, with one end inserted inside and the other outside of a petroleum jelly (Vaseline) well built around the nerve, and amplified with a Differential AC amplifier (A-M Systems 1700). Intracellular recordings were made from the neuronal cell bodies with sharp glass microelectrodes pulled using a Flaming-Brown P-97 micropipette puller (Sutter Instruments) and containing $0.6 \mathrm{M} \mathrm{K}_{2} \mathrm{SO}_{4}$ and $20 \mathrm{~mm} \mathrm{KCl} \mathrm{(final} \mathrm{electrode} \mathrm{resistance} \mathrm{20-30} \mathrm{M} \Omega$ ). All intracellular recordings were performed in single- or two-electrode current-clamp or two-electrode voltage-clamp mode (Axoclamp 2B amplifiers, Molecular Devices). Pyloric neurons are identified according to their characteristic axonal projections in identified nerves and interactions with other STG neurons (diagrammatically shown in Fig. 1 B; Weimann et al., 1991; Blitz et al., 2008).

All data reported here are from PD neurons. Two PD neurons are electrically coupled to each other (Fig. $1 B$ ) and currents were measured in one of the two PD neurons in normal saline without making any attempt to uncouple them. Three currents were measured in two- electrode voltage-clamp mode and leak was subtracted as described before (Khorkova and Golowasch, 2007). (1) A high-threshold potassium current $\left(I_{\mathrm{HTK}}\right)$ was measured as the current activated in normal saline by applying $1000 \mathrm{~ms}$ depolarizing voltage steps from a holding potential of $-40 \mathrm{mV}$. Although this current is composed of two separate currents, a large Ca-dependent $\mathrm{K}^{+}$current and a small delayed rectifier current (Golowasch and Marder, 1992b; Khorkova and Golowasch, 2007), we did not attempt to separate them. (2) A transient A-type current $\left(I_{\mathrm{A}}\right)$, which is fully inactivated at holding voltages of $-40 \mathrm{mV}$, was thus determined by subtraction of $I_{\mathrm{HTK}}$ from the currents measured at the same membrane potentials but from a holding voltage of $-80 \mathrm{mV}$. (3) A hyperpolarization-activated current $\left(I_{\mathrm{H}}\right)$ was activated with 6-s-long hyperpolarizing pulses from a holding potential of $-40 \mathrm{mV}$. Given that typically these currents are measured under conditions in which oscillatory activity from these neurons is blocked [in tetrodotoxin (TTX), for example], controls were performed to make sure that these currents could be faithfully measured in the absence of such a blocker. Figure $2 \mathrm{~A}$ shows the currents measured in normal saline (black traces) with superimposed (red) traces of the same currents measured after $10^{-7}$ M TTX was applied. As can be seen, most current traces overlap to a large degree. This allows us to be confident that the current parameter estimates (see Current fitting and dynamic clamp) obtained from currents measured in normal saline are consistent with those measured under the best conditions possible for this system.

$I_{\text {HTK }}$ is a current composed of several currents under our recording conditions. At least two $\mathrm{K}^{+}$currents are part of it and in normal saline, one $\mathrm{Ca}^{2+}$ and one $\mathrm{Na}^{+}$current also are activated. While the two $\mathrm{K}^{+}$ currents are large, the two inward currents are at least 100 times smaller and contribute $<1 \%$ of the total current. Thus, $I_{\mathrm{HTK}}$ can be thought of a mix of two $\mathrm{K}^{+}$, mostly dominated by a $\mathrm{Ca}^{2+}$-activated component (Golowasch and Marder, 1992b). We did not attempt to separate these two components in this study because coexpression of ion currents in these cells was originally shown for this compound $I_{\mathrm{HTK}}$ (Khorkova and Golowasch, 2007).

Current fitting and dynamic clamp. After we measured the currents described above, we used a manual fitting program that calculates currents using Hodgkin-Huxley-type equations to match voltage clampmeasured currents. The characteristic voltage and kinetic parameters of 
A
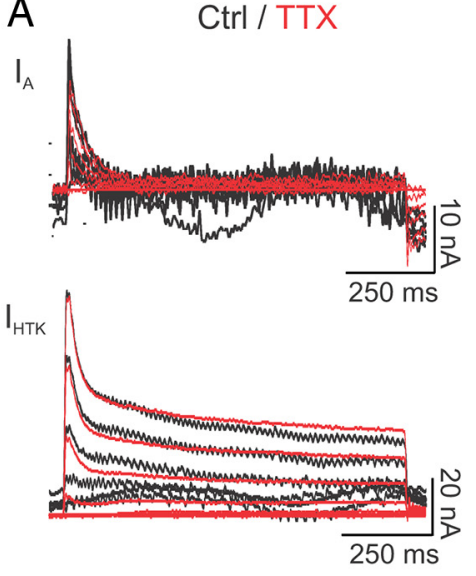

$\mathrm{I}_{\mathrm{H}}$

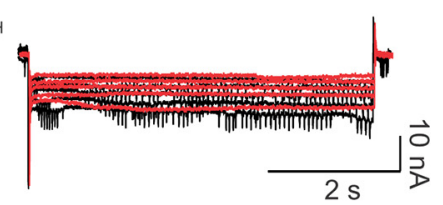

B Raw / Fit
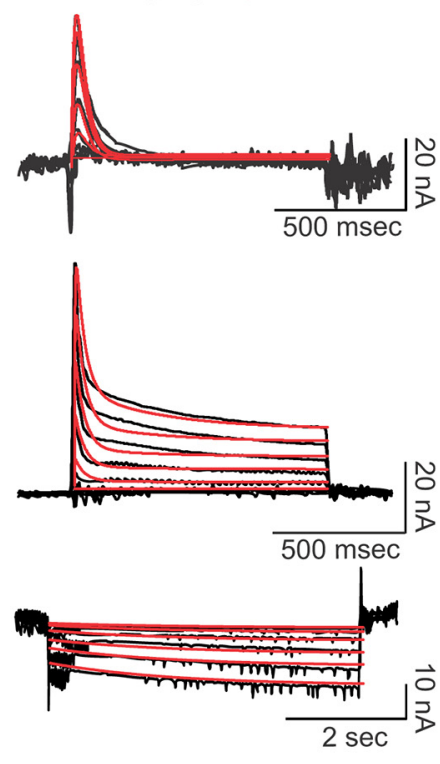

C

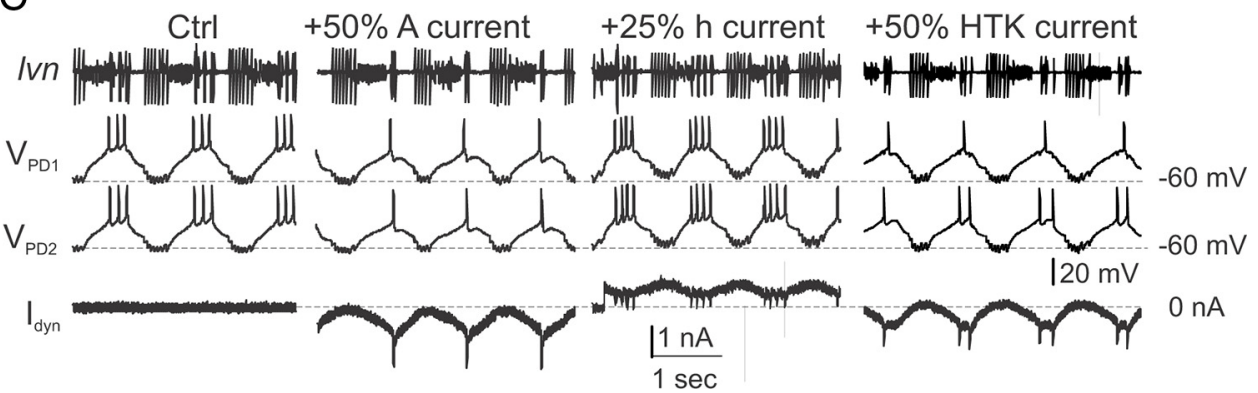

Figure 2. Measurement and fitting of ionic currents. $I_{A}, I_{\mathrm{HTK}}$ and $I_{\mathrm{H}}$ were measured as described in Materials and Methods. $A$, Shown here is one example of the three leak-subtracted currents measured in a single PD neuron. Command voltages for $I_{\mathrm{A}}$ and $I_{\mathrm{HTK}}$ were $-30 \mathrm{to}+20 \mathrm{mV}$, and for $I_{\mathrm{H}}-60$ to $-100 \mathrm{mV}$, in increments of $10 \mathrm{mV}$. Superimposed in red are traces from the same cell after $10^{-7} \mathrm{M} \mathrm{TTX}$ was added to the bath showing only minor differences in the currents recorded under these two conditions. $B$, Current traces from a different PD neuron recorded in normal saline and using the same protocols as in $\boldsymbol{A}$ (black). Superimposed (in red) are fits to the raw current traces with Hodgkin-Huxley-type equations. $\boldsymbol{C}$, Recordings of each of the two PD neurons in a single preparation. Dynamic clamp currents (bottom trace) were calculated using the voltage recording from $\mathrm{PD}_{1}$ to change $G_{A^{\prime}} G_{H^{\prime}}$ and $G_{H T K}$ as indicated, and was injected into both $P D_{1}$ and $P_{2}$ neurons. Note the similarity in the voltage excursions. Top trace is from the Ivn.

these currents can then be used by our dynamic clamp software to modify the intrinsic current levels. The equations used had the form:

$$
\begin{gathered}
I_{\mathrm{x}}(V)=\bar{g}_{1 \mathrm{x}} m_{1}(V) h_{1}(V)\left(V-E_{\mathrm{x}}\right)+\bar{g}_{2 \mathrm{x}} m_{2}(V) h_{2}(V)\left(V-E_{\mathrm{x}}\right), \\
m_{\infty}(V)=\frac{1}{1+\exp \left(-\left(V-V_{\mathrm{m}}\right) / K_{\mathrm{m}}\right)} \\
\frac{d m}{d t}=\frac{m_{\infty}(V)-m}{\tau_{\mathrm{m}}(V)}
\end{gathered}
$$

where $X$ indicates the ionic conductance, $m(V)$ the activation term, and $h(V)$ the inactivation term. For $I_{\mathrm{A}}$ and $I_{\mathrm{HTK}}$, two independent inactivation conductance terms were needed. The activation and inactivation terms have the same form (here written only for $m(V)$ ):

$$
\tau_{\mathrm{m}}(V)=T_{\mathrm{m} \_ \text {low }}+\frac{T_{\mathrm{m} \_ \text {hi }}-T_{\mathrm{m} \_ \text {low }}}{1+\exp \left(-\left(V-V_{\mathrm{m}}\right) / K_{\mathrm{m}}\right)} .
$$

An example of such fits is shown in Figure $2 B$ and examples of the parameters used for the three currents in one of the preparations are listed in Table 1.

The dynamic clamp software we use, available at http://stg.rutgers.edu/ software/, makes use of a NI PCI-6070-E board (National Instruments) on a Windows platform. This system measures the neuronal membrane potential in real time (4 kHz sampling frequency) to calculate an ionic
Table 1. Hodgkin-Huxley equation parameters used in fits shown in Figure 2, and equations in text

\begin{tabular}{lrrrrr}
\hline & $I_{\mathrm{A}}(1)$ & $I_{\mathrm{A}}(2)$ & $I_{\text {HTK }}(1)$ & \multicolumn{1}{c}{$I_{\text {HTK }}(2)$} & \multicolumn{1}{c}{$I_{\mathrm{h}}$} \\
\hline$V_{\mathrm{m}}$ & -15 & 10 & -10 & 20 & -75 \\
$K_{\mathrm{m}}$ & -5 & -15 & -10 & -20 & 50 \\
$V_{\mathrm{h}}$ & -40 & -40 & -25 & 25 & \\
$K_{\mathrm{h}}$ & 10 & 5 & 1 & 20 & \\
$T_{\mathrm{m}}$-low & 1 & 100 & 1 & 1 & 2000 \\
$T_{\mathrm{m}}$-hi & 60 & 100 & 50 & 1 & 2000 \\
$T_{\mathrm{h}}-$-low & 30 & 200 & 5 & 50 & \\
$T_{\mathrm{h}}$-hi & 80 & 400 & 5 & 50 & \\
$G_{\text {max }}(\mathrm{nS})$ & 1500 & 40 & 1000 & 1200 & 150 \\
$E_{\text {rev }}(\mathrm{mV})$ & -80 & -80 & -80 & -80 & -10 \\
\hline & & & & &
\end{tabular}

current that can then be injected into the same neuron (Sharp et al., $1993 \mathrm{a}, \mathrm{b})$. We used this to vary $I_{\mathrm{A}}, I_{\mathrm{HTK}}$, and $I_{\mathrm{H}}$ by modifying their maximal conductances over a range of positive and negative values around the endogenous level of each current $(-50,-25,0,25$, and $50 \%)$. Thus, in any given experiment, 125 different maximum conductance combinations were defined, and the three currents were calculated and injected back into the same PD neuron in two-electrode current-clamp mode. To avoid current flow through gap junctions between the two electrically coupled PD neurons, the same current that was injected into the primary 
PD neuron was injected into the second PD neuron in single-electrode current-clamp mode (Rabbah and Nadim, 2005) after appropriately scaling it using a Brownlee Amplifier (Model 410, Brownlee Precision). In Figure $2 C$ we show the effects of such current injections on both PD neurons. It is clear that this method modifies the membrane potential of both neurons to the same extent.

Recording, analysis, and statistics. Data were acquired using pClamp 9 software (Molecular Devices) or the Scope software (available at http:// stg.rutgers.edu/software), sampled at $4 \mathrm{kHz}$, and saved on a PC using a NI PCI-6070-E data acquisition board (National Instruments). Statistical and graphical analyses were done using Datamaster version 2.2 (available at http://stg.rutgers.edu/software), SigmaPlot 11.0 (Aspire Software), Origin 7.0 (OriginLab), and Matlab R2010b (MathWorks). Reported statistical significance indicates that the significance level $p$ was below $\alpha=0.05$. In this study we characterized activity using 10 properties or attributes (illustrated in Fig. 1C): spike number, spike frequency (= (spike number 1)/burst duration), slow-wave rising slope (calculated between the end of the last IPSP and the first action potential), minimum slow-wave amplitude or "low bound" [ measured as the voltage at end of the last IPSP from the lateral pyloric (LP) neuron], and maximum slow-wave amplitude or "high bound" (measured as the average action potential baseline level), slow-wave amplitude (= high bound - low bound), oscillation period (measured as the time between the first PD neuron action potentials of two consecutive cycles), and PD and LP burst phase onset and end (PDoff, LPon, and LPoff, respectively; with phase defined as the fraction of an oscillation period at which an event occurs). PD onset is used as reference point and is thus always defined as zero phase. Thus, for example, LP onset phase is the fraction of a cycle relative to the beginning of PD burst at which the LP neuron fires the first action potential of a burst.

\section{Results}

As long as neuromodulatory inputs from rostral ganglia to the STG pyloric network of decapod crustaceans are intact (Fig. $1 A$ ), pyloric activity is robust and stereotyped. Pyloric activity depends on the intrinsic properties of each of its neurons as well as the synaptic connections between them (Fig. $1 B$ ) and can be characterized by a number of attributes that can be measured from intracellular somatic as well as extracellular nerve recordings. In this study we characterized activity using 10 attributes: slow-wave rising slope, spike number, spike frequency, maximum slowwave amplitude (high bound) and minimum slow-wave amplitude (low bound), LP burst phase onset and end (LPon, LPoff), PD burst phase end (PDoff), slow-wave amplitude (= high bound - low bound), and oscillation period (Fig. $1 C$, red labels). We examined the effect that correlated changes in the maximum conductance of ionic currents $I_{\mathrm{A}}, I_{\mathrm{H}}$, and $I_{\mathrm{HTK}}$ (defined in Materials and Methods) have on these activity attributes. We hypothesize that, because PD neurons from different animals under control conditions express these currents in a linearly correlated manner (Schulz et al., 2006; Khorkova and Golowasch, 2007; Temporal et al., 2012), at least a subset of activity attributes should be determined by pairs or triplets of these currents. Furthermore, pairs or triplets of conductance values should exist for which these activity attributes remain invariant (referred to as level sets). In particular, we hypothesize that the known invariance of phase relationships between PD and LP neurons in the network (Bucher et al., 2005; Goaillard et al., 2009) depends on these correlations.

For every experiment we first measured the 10 activity attributes in the unstimulated (unperturbed) system (Table 2). We then recorded the three voltage-gated currents $\left(I_{\mathrm{A}}, I_{\mathrm{HTK}}\right.$, and $\left.I_{\mathrm{H}}\right)$ and immediately fitted them with Hodgkin-Huxley-type equations (Fig. $2 \mathrm{~B}$ ). To obtain reasonably close fits, both $I_{\mathrm{A}}$ and $I_{\mathrm{HTK}}$ required two voltage-gated components, each consisting of one activation and one inactivation term. The equations and param-
Table 2. Activity attributes in the unstimulated system

\begin{tabular}{lcll}
\hline Attribute & Average & SD & CV \\
\hline Slope $(\mathrm{mV} / \mathrm{s})$ & 0.094 & 0.048 & 0.511 \\
Spike number & 5.7 & 1.19 & 0.209 \\
Spike frequency $(\mathrm{Hz})$ & 29.0 & 8.9 & 0.308 \\
High bound $(\mathrm{mV})$ & -40.4 & 4.5 & 0.111 \\
Low bound $(\mathrm{mV})$ & -57.1 & 5.3 & 0.093 \\
LPon & 0.39 & 0.08 & 0.205 \\
LPoff & 0.69 & 0.08 & 0.116 \\
PDoff & 0.22 & 0.038 & 0.173 \\
Amplitude $(\mathrm{mV})$ & 16.8 & 2.9 & 0.173 \\
Period (Hz) & 0.83 & 0.27 & 0.325 \\
\hline
\end{tabular}

Except for period and LP phases, attributes correspond to those of PD neurons $(n=20)$.

eters used for the currents in Figure $2 B$ are shown in Materials and Methods. These fits vary in quality from cell to cell, with one current or another producing a more accurate fit. This is due in part to the fact that these currents had to be measured in normal saline, in contrast with standard practice in which rhythmic activity of the pyloric network is blocked, by bath application of tetrodotoxin (Khorkova and Golowasch, 2007), or by blocking action potential transmission along the stomatogastric nerve, which removes neuromodulatory input from central ganglia (CoGs and OG, Fig. $1 A$ ) necessary for enabling rhythmic activity (Thoby-Brisson and Simmers, 1998; Luther et al., 2003). Measuring ionic currents when the network is rhythmically active unavoidably introduces some degree of error to these measurements (Graubard and Hartline, 1991; Golowasch and Marder, 1992b). However, after confirming that the current recordings obtained in normal saline were very similar to those obtained immediately after $10^{-7} \mathrm{M}$ TTX was applied (Fig. 2A), we deemed such error in the fits to be acceptable under these conditions.

\section{Ionic current correlations determine activity attributes}

After measuring and fitting the three currents, a matrix was built with maximum conductance values of each of the three currents set at $-50,-25,0$ (endogenous level), 25, and 50\% level relative to the endogenous value, thus generating 125 maximum conductance combinations. Currents in each one of these combinations were subsequently injected back, using dynamic clamp, into the same PD neuron from which they were measured as well as the second PD neuron to ensure that the membrane potential of both PD neurons changed to the same extent (Fig. 2C). The activity of the PD neuron and of the entire pyloric network was recorded for each combination and stored for analysis. The 10 activity attributes were later measured and the difference, relative to control, was calculated. Henceforth, we report the percentage of change in activity attribute as a function of the percentage of change in the dynamic clamp-injected ionic conductance.

Subsequent analysis was performed in the four-dimensional (4-D) space created by varying the three conductances and measuring each activity attribute. However, to illustrate the general features of this analysis, Figure 3 shows an example of the changes observed in spiking frequency with respect to pairwise changes of ionic conductances, while keeping the third ionic conductance at its control level ( $0 \%$ change). In this example we determined the 2-D planes that best fit the observed spiking frequency changes using a multivariate linear regression with the plane described by $Z=\beta_{\mathrm{X}} A_{\mathrm{X}}+\beta_{\mathrm{Y}} A_{\mathrm{Y}}+\beta_{0}$ (Fig. 3, left). The resulting coefficients $\beta_{\mathrm{X}}$ and $\beta_{\mathrm{Y}}$ indicate the strength and direction of the spiking frequency changes as a function of the conductance changes shown on the $X$-and $Y$-axes, respectively. Thus, in the case illustrated in Figure $3 A$, the maximum conductance of $I_{\mathrm{A}}$ was kept at its control 
value and the maximum conductances of $I_{\mathrm{HTK}}$ and $I_{\mathrm{H}}\left(G_{\mathrm{HTK}}, G_{\mathrm{H}}\right)$ were varied. The corresponding coefficients were $\beta_{\mathrm{HTK}}=-0.363$ and $\beta_{\mathrm{H}}=0.869$, with a highly significant degree of correlation of spiking frequency changes with these two conductances $(p<0.001, n=11)$ and a coefficient of determination $R^{2}=0.568$. These results indicate that spiking frequency is positively correlated with $G_{\mathrm{H}}$ changes and negatively correlated with $G_{\text {HTK }}$ changes. The coefficient of determination and correlation coefficients further indicate that variations of these two currents account for $\sim 57 \%$ of the observed variance, and that spiking frequency is 2.4 times more sensitive to $G_{\mathrm{H}}$ than to $G_{\mathrm{HTK}}$ changes. Following the same line of analysis, we observed that spiking frequency negatively depends on changes of both $G_{\mathrm{A}}$ and $G_{\mathrm{HTK}}$, with $G_{\mathrm{H}}$ constant at control level $\left(\beta_{\mathrm{A}}=-0.601, \beta_{\mathrm{HTK}}=-0.645, p<\right.$ $0.001, R^{2}=0.422, n=11$; Fig. $3 B$, left). Finally, Figure $3 C$ (left) shows the relationship for the third pair of conductances confirming a negative dependence of spiking frequency on changes of $G_{\mathrm{A}}$ and positive dependence on changes of $G_{\mathrm{H}}$ $\left(\beta_{\mathrm{A}}=-0.476, \beta_{\mathrm{H}}=0.926 ; R^{2}=0.579\right.$; $p<0.001, n=11)$. Overall, these data indicate that spiking frequency is most dependent on $G_{\mathrm{H}}$, and somewhat less sensitive to both $G_{\mathrm{A}}$ and $G_{\mathrm{HTK}}$.

These linear relationships in two dimensions reveal that a set of pairs of conductance values exists for which the activity attribute remains invariant at any given value (a level set). By projecting the planes obtained from the fits shown in Figure 3 onto two dimensions and using a colorscale to represent the changes in activity, such a set of conductance pairs can be visualized (Fig. 3, right). A black dashed line in each of the panels illustrates the level set for the spiking frequency observed in control conditions (spiking frequency change $=0 \%$ ) that was calculated from the multivariate linear regression equation by setting $Z=0$. As seen in this example, positive correlations between $G_{\mathrm{H}}$ and either $I_{\mathrm{HTK}}$ (Fig. $3 A$, right) and $G_{\mathrm{A}}$ (Fig. $3 C$, right), and a negative correlation between the two potassium conductances (Fig. 3B, right) are conditions for the invariance of spiking frequency in PD neurons. Furthermore, these data indicate that spiking frequency is most sensitively dependent on $G_{\mathrm{H}}$ (largest $\beta$ value) and similarly sensitive to both $G_{\mathrm{A}}$ and $G_{\mathrm{HTK}}$.

We performed the same analysis on each of the 10 activity attributes measured, this time using a four-dimensional multivariate analysis (with $Z=\beta_{\mathrm{A}} X_{\mathrm{A}}+\beta_{\mathrm{H}} X_{\mathrm{H}}+\beta_{\mathrm{HTK}} X_{\mathrm{HTK}}+\beta_{0}$ ), with the three conductances as independent variables for each activity attribute. The results are shown in Table 3 and summarized in Figure 4. The top row in Figure 4 shows the signs of the coefficients after setting $Z=$ constant $=0$ (meaning no change from control values) and solving for one of the conductances. The signs (positive or negative) then indicate the direction of relative change between two conductances for which activity remains invariant. The variations of the conductances examined define four conductance relationship categories that affect spe- cific activity attributes (Fig. 4, top). (1) All negative coefficients correspond to the case where a change in any single conductance is opposed by each of the other two conductances (Fig. 4A). A single activity attribute, slow-wave amplitude, follows this pattern. (2) The $G_{\mathrm{H}}$ coefficient shows a positive (or negative) sign and the two potassium conductances have the opposite sign, corresponding to the case where both potassium currents oppose the effects of changes in $I_{\mathrm{H}}$ (Fig. $4 \mathrm{~B}$ ). To this category belong most activity attributes examined: slope of the rising phase, spike number and spike frequency, the high and low levels of the slow wave (high bound and low bound), and two of the three measured phase relationships between PD and LP neuron activity (PDoff and LPoff). (3) Only one pair of currents $\left(I_{\mathrm{A}}\right.$ and $\left.I_{\mathrm{H}}\right)$ affects an activity attribute, namely LPon, and both need to change in the same direction to keep this attribute constant (Fig. 4C). (4) The final category corresponds to that in which no relationships between a pair or triplet of conductances are statistically significant in altering activity attributes. The pyloric rhythm period is the one attribute that falls in this category (Fig. 4D).

Overall, these results indicate that all activity attributes, but one, of PD neurons, or of the pyloric network, are determined by combinations of at least two of the ionic currents examined, and most by all three. Furthermore, except for one (amplitude), all of these combinations reflect compensatory effects of one current by the others. Interestingly, most activity attributes are determined by positive relationships between $G_{\mathrm{H}}$ and the two $\mathrm{K}^{+}$ 
Table 3. Coefficients from multivariate linear regression analysis $\left(Z=\beta_{A} X_{A}+\right.$ $\boldsymbol{\beta}_{\mathrm{H}} X_{\mathrm{H}}+\boldsymbol{\beta}_{\mathrm{HTK}} X_{\mathrm{HTK}}+\boldsymbol{\beta}_{0}$ ) of each of the 10 activity attributes measured in this study

\begin{tabular}{llrlll}
\hline Attribute & \multicolumn{1}{l}{$\beta_{\mathrm{A}}$} & \multicolumn{1}{l}{$\beta_{\mathrm{H}}$} & \multicolumn{1}{l}{$\beta_{\mathrm{HTK}}$} & $R^{2}$ & \multicolumn{1}{l}{$\beta_{\mathrm{A}}+\beta_{\mathrm{H}}+\beta_{\mathrm{HTK}}$} \\
\hline Slope & $-0.746^{* * *}$ & $1.826^{* * *}$ & $-1.127^{* * *}$ & 0.764 & -0.047 \\
Spike number & $-0.604^{* * *}$ & $1.222^{* * *}$ & $-0.721^{* * *}$ & 0.596 & -0.101 \\
High bound & $0.138^{* * *}$ & $-0.206^{* * *}$ & $0.170^{* * *}$ & 0.465 & 0.102 \\
Low bound & $0.019^{* *}$ & $-0.322^{* * *}$ & $0.027^{* * *}$ & 0.721 & -0.283 \\
LPoff & $-0.066^{* * *}$ & $0.551^{* * *}$ & $-0.044^{* *}$ & 0.705 & 0.441 \\
Spike frequency & $-0.565^{* * *}$ & $0.781^{* * *}$ & $-0.748^{* * *}$ & 0.503 & -0.532 \\
LPon & $-0.084^{* *}$ & $0.784^{* * *}$ & -0.049 & 0.572 & 0.651 \\
PDoff & $-0.477^{* * *}$ & $1.430^{* * *}$ & $-0.092^{*}$ & 0.649 & 0.861 \\
Amplitude & $-0.214^{* * *}$ & $-0.605^{* *}$ & $-0.304^{* * *}$ & 0.712 & -1.123 \\
Period & 0.033 & $-1.866^{* * *}$ & 0.070 & 0.491 & -1.763
\end{tabular}

${ }^{*} p<0.05,{ }^{* *} p<0.01,{ }^{* * *} p<0.001 . N=11 . R^{2}$ from the linear fits indicates the amount of variance explained by these relationships.

conductances (Fig. $4 B$ ) as our experimental results (Khorkova and Golowasch, 2007) suggest. However, $G_{\mathrm{A}}$ and $G_{\mathrm{HTK}}$ in this category show a negative correlation, indicating that the two conductances tend to compensate for each other, in contrast to our previous experimental results, which showed a positive correlation between the two (Khorkova and Golowasch, 2007). The fact that, together, $G_{\mathrm{A}}$ and $G_{\mathrm{HTK}}$ appear to positively and linearly compensate for changes in $G_{\mathrm{H}}$ in this category is further suggested by looking at the sum of the coefficients that determine the direction and strength of these effects (Table 3, last column). If $G_{\mathrm{H}}$ was compensated for exactly by the other two conductances, the sum of the three conductances should be zero. The sum of $\beta_{\mathrm{A}}$ $+\beta_{\mathrm{H}}+\beta_{\mathrm{HTK}}$ in Table 3 shows that 6 of the 7 attributes in this category have small or moderate deviations (positive or negative) from zero, suggesting that positive correlations between $G_{\mathrm{H}}$ and the $\mathrm{K}^{+}$currents (but negative correlations between the $\mathrm{K}^{+}$currents) are best at balancing out and thus maintaining those activity attributes relatively invariant.

\section{$I_{H}$ and positive conductance correlations define invariance of most activity attributes}

To estimate the relative contributions of each ionic current to the various activity attributes we normalized the slope coefficients and compared their amplitudes. We did this by dividing each coefficient value from the multivariate analysis by the sum of the absolute values of the corresponding three coefficients for each activity attribute (from Table 3 ). These normalized coefficient values are shown in Figure 5. One clear pattern emerges from this picture: $I_{\mathrm{H}}$ is the dominant current in specifying every measured activity attribute. In several instances, this dominance is subtle (slope, spike number, spike frequency, and high bound), but in others it is very strong (LPon, LPoff, PDoff, period). Interestingly, $I_{\mathrm{H}}$ is the only current that significantly affects period (Table $3)$. In the case of LPon, $I_{\mathrm{H}}$ and $I_{\mathrm{A}}$ control it in a homeostatic manner (they oppose each other), even though $I_{\mathrm{H}}$ is clearly stronger, and $I_{\text {HTK }}$ has no significant influence (Table 3 ). For all remaining activity attributes, except amplitude, the effect of $I_{\mathrm{H}}$, whether positive or negative, is always counteracted by the two potassium currents, with the effects nearly completely cancelling each other out for the slope, spike number and high bound attributes (Table 3 ).

If linear relationships among the three currents influence most of the attributes that characterize the activity of PD neurons, we hypothesize that the fraction of the variance accounted for by the interactions between these currents (reflected in the coefficient of determination, $R^{2}$, listed in Table 3 ) might be re- lated to the natural variance of these activity attributes in the unperturbed system. To test this hypothesis, we calculated the coefficient of variation $(\mathrm{CV})$ of each of the 10 attributes in the free running system before any ionic currents were measured or injected back into the cells with dynamic clamp (Table 2) and used these values as a measure of the natural variability of the system. For this, we recorded extracellularly from both the lateral ventricular and pyloric dilator nerves and from the PD neuron with a single electrode the first time it was impaled (to minimize the disruption of its activity). On the other hand, since $R^{2}$ represents the fraction of the variance explained by the independent variables in the 4 -D multivariate linear analysis, $1-R^{2}$ represents the unexplained portion of the variance, and can thus be an indicator of variability of the dynamic clamp-perturbed system (Fig. 6). Interestingly, we observe that the variability of the activity attributes in the minimally perturbed system shows a high correlation with the unexplained variance determined from the multivariate analysis of the dynamic clamp-perturbed system $(\rho=0.769, p<$ 0.01 , Pearson Product moment correlation test). We conclude from this that the portion of the variance of the activity attributes that can be explained by changes in the three ionic currents examined here are also at play, and to a similar degree, in the free running and undisturbed system.

\section{Discussion}

Correlations between ionic currents have been discovered in several systems (Linsdell and Moody, 1994; MacLean et al., 2003; Vähäsöyrinki et al., 2006; Khorkova and Golowasch, 2007; Peng and $\mathrm{Wu}, 2007$; Tobin et al., 2009; Bergquist et al., 2010; Cao and Oertel, 2011; Amendola et al., 2012). This interdependence suggests compensatory roles between the correlated conductances in determining specific attributes of neuronal or network activity, and may be of fundamental functional importance given the high levels of individual conductance variability observed in identified neurons across individuals (Golowasch et al., 1999; Liss et al., 2001; Schulz et al., 2006; Khorkova and Golowasch, 2007; Olypher and Calabrese, 2007; Temporal et al., 2012). Here we examined experimentally the extent to which compensatory relationships among three ionic currents, $I_{\mathrm{A}}, I_{\mathrm{H}}$, and $I_{\mathrm{HTK}}$, may be able to specify invariance in a number of activity attributes in an identified neuron from the crab pyloric network. We conclude that, indeed, correlated changes in ionic conductances define sets of conductance pairs or triplets that, together, can control activity attributes by specifying invariant levels of activity (level sets) along linear combinations of maximal conductances. Up to $\sim 76 \%$ of the variance of some attributes can be explained with changes in only these three currents (Table 3). Given that conductance variability and the correlated expression of ionic currents appear to be prevalent phenomena in neurons from different systems, this suggests a general mechanism to ensure stability of activity despite the variable expression of ionic currents.

It is interesting that, to date, only what appears to be linear relationships between ion current parameters have been identified experimentally (Schulz et al., 2006, 2007; Khorkova and Golowasch, 2007; Tobin et al., 2009; Cao and Oertel, 2011; Amendola et al., 2012; Temporal et al., 2012), while theoretically, linear relationships appear to be the exception (Golowasch et al., 2002; Olypher and Calabrese, 2007; Taylor et al., 2009; Hudson and Prinz, 2010) and appropriate only locally (Olypher and Calabrese, 2007). Also, the ranges over which these currents, their conductances, or the levels of mRNA that code for them vary linearly are very large (2- to 10 -fold), much larger than what 

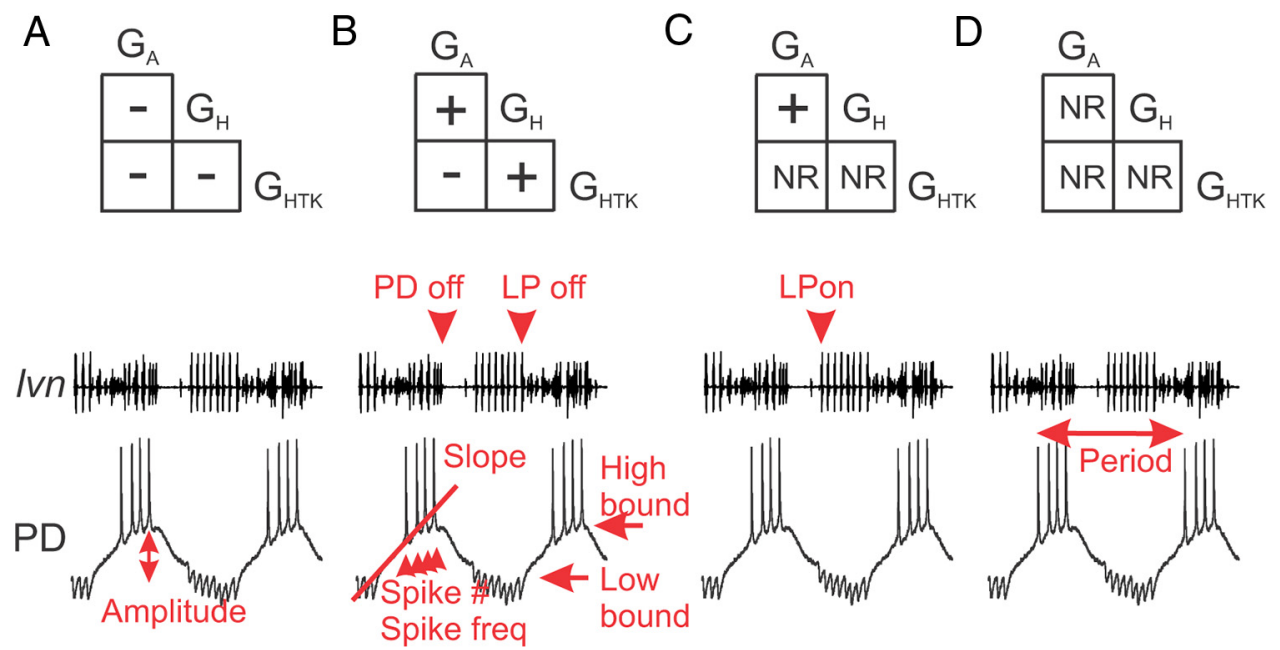

Figure 4. Relationship among $G_{A^{\prime}} G_{H T K}$ and $G_{H}$ define distinct attributes. Coefficients derived from the multivariate linear analysis $\left(Z=\beta_{A} X_{A}+\beta_{H} X_{H}+\beta_{H T K} X_{H T K}+\beta_{0}\right)$ listed in Table 3 define four activity attribute categories $(\boldsymbol{A}-\boldsymbol{D})$ relative to the correlated variation of the ionic conductances examined. Top shows the conductance relationships that determine these categories; bottom graphically shows the activity attributes in each category. A positive sign means that to maintain constant at attribute value (e.g., at control levels, corresponding to $Z=0$ ), currents must grow together; a negative sign means that attribute invariance requires a current to grow and the other to decrease.

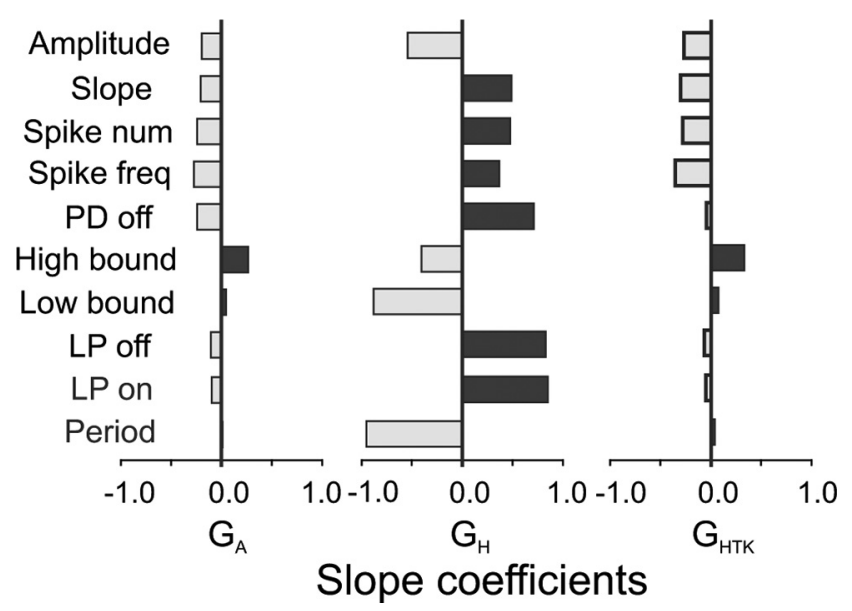

Figure 5. $G_{H}$ dominates over the potassium conductances in their influence on all activity attributes in PD neurons. Each ionic conductance coefficient (derived from the 4-D multivariate linear analysis) is plotted as a (signed) fraction of the total. Each normalized coefficient was calculated by dividing its raw value by the sum of the absolute values of the three coefficients for each activity attribute (listed on the left). Different in-bar fills are simply to highlight differences in the sign of the coefficients.

might be considered local. We examined similar ranges here ( $\pm 50 \%$, corresponding to a threefold change) and also found that linear relationships among them can specify invariant activity features. Nevertheless, mild nonlinearities may be obscured by measurement error and noise. In any case, the mechanisms that allow such relationships between conductance levels are yet to be elucidated.

How can compensatory changes of specific activity features be mediated by ionic currents that are both not linearly dependent on parameters (e.g., voltage or $\mathrm{Ca}^{2+}$ ) and activated over voltage ranges that sometimes barely overlap (e.g., $I_{\mathrm{A}}$ and $I_{\mathrm{H}}$ )? One possibility is that only the steady-state portion of these currents over only very narrow voltage ranges are essential and may affect activity indirectly by regulating the resting potential or leak current at that voltage (Cao and Oertel, 2011). Alternatively, complex interactions of voltage-dependent gating variables and rate constants combine to stabilize a feature (Olarinre et al., 2012).

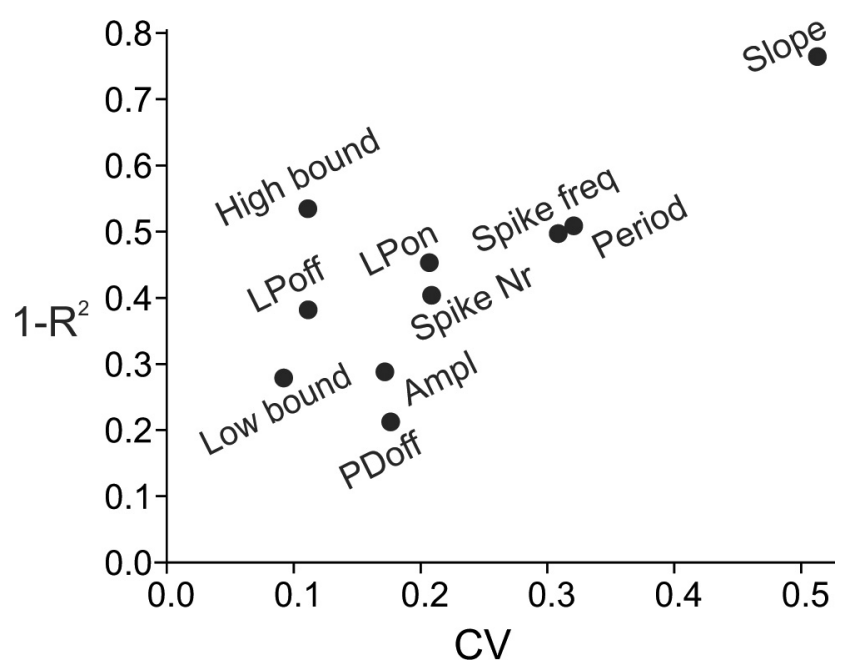

Figure 6. The variances of the activity of the unperturbed and the dynamic clamp-injected PD neuron closely match. The CV of the unperturbed neurons $(N=20)$ and the mean unexplained variance $\left(1-R^{2}\right)$ of the dynamic clamp-perturbed PD neurons (from multivariate linear analysis, $N=11$ ) for each activity attribute are plotted against each other. Except for deviations observed at low levels of variability, these two sources of variation appear to be closely correlated with each other $(\rho=0.769, p=0.009$, Pearson Product moment correlation test).

The variance of some attributes, which are not well accounted for on the basis of the small number of currents examined in this study, is likely better explained if additional currents expressed by pyloric neurons (Graubard and Hartline, 1991; Golowasch and Marder, 1992a; Zhang et al., 1995) are included. Indeed, Olypher and Calabrese (2007) have shown proof that to maintain $m$ features of activity constant, at least $m+1$ independent compensating parameters are required. Consequently, we cannot explain activity constancy of more than two features with the three currents we are considering. This may explain the partial levels of variance that we can account for in the measured features.

To our knowledge, except for two cases (Bergquist et al., 2010; Ransdell et al., 2012), all conductance correlation relationships thus far reported in biological neurons, whether from voltageclamp conductance measurements (MacLean et al., 2003; Tobin 
et al., 2009) or mRNA quantification (Schulz et al., 2006; Goaillard et al., 2009; Tobin et al., 2009), are positive, including those observed in the neuron examined in this study (Khorkova and Golowasch, 2007; Schulz et al., 2007; Temporal et al., 2012). This is in contrast to negative relationships that are typically expected from theoretical studies (Taylor et al., 2009; Hudson and Prinz, 2010). We propose that the positive correlations observed in identified neurons across individuals reflect genetically encoded developmental programs aimed at globally scaling ionic conductances during growth and neuronal-type specification. The positive correlations observed in adults would thus correspond to an ontogenically preserved state. Pairs of currents that are functionally inadequate to control these activity attributes (e.g., $G_{\mathrm{A}}-G_{\mathrm{HTK}}$ ) could be regulated with a negative relationship by other mechanisms, such as activity-dependent conductance changes. In fact, activity-dependent mechanisms have been reported in crab pyloric network, particularly for the $G_{\mathrm{A}}-G_{\mathrm{HTK}}$ conductance pair, albeit in a different neuron type (Golowasch et al., 1999). Our work then predicts that negative conductance relationships, such as those observed in our study and perhaps others predicted by theory, should be observed in future studies, and that they are driven by short- to medium-term induction mechanisms such as activity dependence or neuromodulatory inputs. Hudson and Prinz (2010), using generic models of STG neurons, reported that $30 \%$ of correlations that contribute to maintaining neuronal activity attributes invariant are negative. One of those negative relationships was also observed in our study: a negative $G_{\mathrm{A}}-G_{\mathrm{HTK}}$ relationship accounts for their duty cycle values, which is closely related to our PDoff attribute. Given that these two $\mathrm{K}^{+}$ conductances are transient and activate in a similar voltage range (Golowasch and Marder, 1992b), this relationship should not be surprising. These authors also found several other relationships that we did not attempt to study, but which are interesting candidates for future studies.

All phase relationships of burst firing in the pyloric network (Bucher et al., 2005; Goaillard et al., 2009) are essentially constant across individuals. Although the phase of PD onset is typically not reported because it is used as reference, if measured with respect to LP burst termination (PDon $=1-$ LPoff), it too would remain invariant given that LPoff is invariant. PDon relative to LP burst termination would be directly related to the slope attribute. Therefore, we conclude that our results are consistent with the idea that the constancy in the natural state of all the phase relationships between PD and LP bursting (Bucher et al., 2005; Goaillard et al., 2009) is likely to be at least partly due to the apparent correlation between $I_{\mathrm{H}}$ and the $\mathrm{K}^{+}$currents $I_{\mathrm{A}}$ and $I_{\mathrm{HTK}}$. Furthermore, our observation that a positive $G_{\mathrm{A}}-G_{\mathrm{H}}$ relationship accounts for an invariant slope (or delay to burst onset after inhibition) is consistent with theoretical and experimental observations by Hudson and Prinz (2010) and others (MacLean et al., 2003, 2005; Burdakov, 2005).

To what extent do the conductance relationships examined here account for changes in activity in the natural, unperturbed and free running state? We looked at this by comparing the coefficient of variation of each of the activity attributes in the unperturbed state to the unexplained portion of the variance $\left(1-R^{2}\right)$ of these attributes obtained from the dynamic clamp experiments (Fig. 6), and observed a clear correlation between these two forms of variability. This suggests that the level of variability in the unperturbed state can be accounted for by changes in the three conductances to an extent similar to that accounted for in our dynamic clamp experiments. Thus, a small CV value for the PDoff attribute, for example, corresponds to a small unexplained variance from the dynamic clamp experiments. In contrast, the variability of the slope attribute, while sensitive to the correlated changes of the three currents, cannot be accounted for to the same extent as, for example, PDoff, suggesting that currents other than those examined in this study affect it to a significant degree as well.

Simultaneous invariance of all activity attributes can obviously not be achieved in any single neuron or neuronal type. Different levels of invariance will be achieved if current relationships (level sets) for each activity attribute differ from each other to varying degrees. Therefore, for any given neuronal type, a hierarchy of attributes will always exist, for which a given set of correlated currents determines their invariance level. For PD neurons, this is reflected, for example, in the rank of the variance of each attribute that is explained by the correlated expression of these currents shown in Figure 6. Interestingly, period, which is known to be extremely variable in this system (Bucher et al., 2005; Goaillard et al., 2009), is near the lowest point in the hierarchy, while the phase relationships, which are the most invariant of these attributes (Bucher et al., 2005; Goaillard et al., 2009), are near the top. The exact origin of the variance difference between attributes remains to be discovered, and may be, at least in part, due to the small number of parameters (ionic conductances) considered in this study, as suggested by Olypher and Calabrese (2007).

\section{References}

Amendola J, Woodhouse A, Martin-Eauclaire MF, Goaillard JM (2012) $\mathrm{Ca}(2)(+) /$ cAMP-sensitive covariation of $I(\mathrm{~A})$ and $I(\mathrm{H})$ voltage dependences tunes rebound firing in dopaminergic neurons. J Neurosci 32:2166-2181.

Bergquist S, Dickman DK, Davis GW (2010) A hierarchy of cell intrinsic and target-derived homeostatic signaling. Neuron 66:220-234.

Blitz DM, White RS, Saideman SR, Cook A, Christie AE, Nadim F, Nusbaum MP (2008) A newly identified extrinsic input triggers a distinct gastric mill rhythm via activation of modulatory projection neurons. J Exp Biol 211:1000-1011.

Bucher D, Prinz AA, Marder E (2005) Animal-to-animal variability in motor pattern production in adults and during growth. J Neurosci 25:1611-1619.

Burdakov D (2005) Gain control by concerted changes in $I(\mathrm{~A})$ and $I(\mathrm{H})$ conductances. Neural Comput 17:991-995.

Cao XJ, Oertel D (2011) The magnitudes of hyperpolarization-activated and low-voltage-activated potassium currents vary in neurons of the ventral cochlear nucleus. J Neurophysiol 106:630-640.

Goaillard JM, Taylor AL, Schulz DJ, Marder E (2009) Functional consequences of animal-to-animal variation in circuit parameters. Nat Neurosci 12:1424-1430.

Golowasch J, Marder E (1992a) Proctolin activates an inward current whose voltage dependence is modified by extracellular $\mathrm{Ca}^{2+}$. J Neurosci 12:810-817.

Golowasch J, Marder E (1992b) Ionic currents of the lateral pyloric neuron of the stomatogastric ganglion of the crab. J Neurophysiol 67:318-331.

Golowasch J, Abbott LF, Marder E (1999) Activity-dependent regulation of potassium currents in an identified neuron of the stomatogastric ganglion of the crab Cancer borealis. J Neurosci 19:RC33(1-5).

Golowasch J, Goldman MS, Abbott LF, Marder E (2002) Failure of averaging in the construction of a conductance-based neuron model. J Neurophysiol 87:1129-1131.

Graubard K, Hartline DK (1991) Voltage clamp analysis of intact stomatogastric neurons. Brain Res 557:241-254

Hudson AE, Prinz AA (2010) Conductance ratios and cellular identity. PLoS Comput Biol 6:e1000838.

Khorkova O, Golowasch J (2007) Neuromodulators, not activity, control coordinated expression of ionic currents. J Neurosci 27:8709-8718.

Linsdell P, Moody WJ (1994) Na + channel mis-expression accelerates K+ channel development in embryonic Xenopus laevis skeletal muscle. J Physiol 480:405-410.

Liss B, Franz O, Sewing S, Bruns R, Neuhoff H, Roeper J (2001) Tuning pace- 
maker frequency of individual dopaminergic neurons by $\mathrm{Kv} 4.3 \mathrm{~L}$ and KChip3.1 transcription. EMBO J 20:5715-5724.

Luther JA, Robie AA, Yarotsky J, Reina C, Marder E, Golowasch J (2003) Episodic bouts of activity accompany recovery of rhythmic output by a neuromodulator- and activity-deprived adult neural network. J Neurophysiol 90:2720-2730.

MacLean JN, Zhang Y, Johnson BR, Harris-Warrick RM (2003) Activityindependent homeostasis in rhythmically active neurons. Neuron 37:109-120.

MacLean JN, Zhang Y, Goeritz ML, Casey R, Oliva R, Guckenheimer J, Harris-Warrick RM (2005) Activity-independent co-regulation of IA and Ih in rhythmically active neurons. J Neurophysiol 94:3601-3617.

Olarinre M, Rotstein H, Golowasch J (2012) A modeling study of conductance co-regulation in neuronal models. Abstr Front Applied Comp Math, FACM 2012:52.

Olypher AV, Calabrese RL (2007) Using constraints on neuronal activity to reveal compensatory changes in neuronal parameters. J Neurophysiol 98:3749-3758.

Peng IF, Wu CF (2007) Drosophila cacophony channels: a major mediator of neuronal $\mathrm{Ca}^{2+}$ currents and a trigger for $\mathrm{K}+$ channel homeostatic regulation. J Neurosci 27:1072-1081.

Rabbah P, Nadim F (2005) Synaptic dynamics do not determine proper phase of activity in a central pattern generator. J Neurosci 25:11269-11278.

Ransdell JL, Nair SS, Schulz DJ (2012) Rapid homeostatic plasticity of intrinsic excitability in a central pattern generator network stabilizes functional neural network output. J Neurosci 32:9649-9658.

Schulz DJ, Goaillard JM, Marder E (2006) Variable channel expression in identified single and electrically coupled neurons in different animals. Nat Neurosci 9:356-362.

Schulz DJ, Goaillard JM, Marder EE (2007) Quantitative expression profiling of identified neurons reveals cell-specific constraints on highly variable levels of gene expression. Proc Natl Acad Sci U S A 104:13187-13191.

Selverston AI, Russell DF, Miller JP (1976) The stomatogastric nervous sys- tem: structure and function of a small neural network. Prog Neurobiol 7:215-290.

Sharp AA, O’Neil MB, Abbott LF, Marder E (1993a) The dynamic clamp: artificial conductances in biological neurons. Trends Neurosci 16:389-394.

Sharp AA, O’Neil MB, Abbott LF, Marder E (1993b) Dynamic clamp: computer-generated conductances in real neurons. J Neurophysiol 69:992-995.

Swensen AM, Bean BP (2005) Robustness of burst firing in dissociated Purkinje neurons with acute or long-term reductions in sodium conductance. J Neurosci 25:3509-3520.

Taylor AL, Goaillard JM, Marder E (2009) How multiple conductances determine electrophysiological properties in a multicompartment model. J Neurosci 29:5573-5586.

Temporal S, Desai M, Khorkova O, Varghese G, Dai A, Schulz DJ, Golowasch J (2012) Neuromodulation independently determines correlated channel expression and conductance levels in motor neurons of the stomatogastric ganglion J Neurophysiol 107:718-727.

Thoby-Brisson M, Simmers J (1998) Neuromodulatory inputs maintain expression of a lobster motor pattern-generating network in a modulationdependent state: evidence from long-term decentralization in vitro. J Neurosci 18:2212-2225.

Tobin AE, Cruz-Bermúdez ND, Marder E, Schulz DJ (2009) Correlations in ion channel mRNA in rhythmically active neurons. PLoS One 4:e6742.

Vähäsöyrinki M, Niven JE, Hardie RC, Weckström M, Juusola M (2006) Robustness of neural coding in Drosophila photoreceptors in the absence of slow delayed rectifier K+ channels. J Neurosci 26:2652-2660.

Weimann JM, Meyrand P, Marder E (1991) Neurons that form multiple pattern generators: identification and multiple activity patterns of gastric/pyloric neurons in the crab stomatogastric system. J Neurophysiol 65:111-122.

Zhang B, Wootton JF, Harris-Warrick RM (1995) Calcium-dependent plateau potentials in a crab stomatogastric ganglion motor neuron. II. Calcium-activated slow inward current. J Neurophysiol 74:1938-1946. 